\title{
Projective subdynamics and universal shifts
}

\author{
Pierre Guillon ${ }^{12} \|^{\mid}$ \\ ${ }^{1}$ CMM, Universidad de Chile \\ ${ }^{2}$ CNRS \& IML, Marseille, France
}

We study the projective subdynamics of two-dimensional shifts of finite type, which is the set of one-dimensional configurations that appear as columns in them. We prove that a large class of one-dimensional shifts can be obtained as such, namely the effective subshifts which contain positive-entropy sofic subshifts. The proof involves some simple notions of simulation that may be of interest for other constructions. As an example, it allows us to prove the undecidability of all non-trivial properties of projective subdynamics.

Keywords: multidimensional symbolic dynamics, effective dynamics, tilings, simulation, undecidability

\section{Introduction}

Computation in dynamical systems has shown an increasing interest in the last decade. One of the questions that arises is the computational power of some models defined dynamically, where the computation result is seen as the "trace" of the system evolution or (equivalently) as a smaller system that it dynamically simulates. For cellular automata, this can be the limit set [Hur87, Maa95] or the column factors [Kůr97, CFG07]. For general effective dynamical systems, this can be observation problems with respect to some partitions [DKB05].

The setting of multidimensional symbolic dynamics is one of the most natural and elegant models with full computational power, as suggested by more recent results [Hoc09a, DRS10, AS10]. These works can be interpreted both as taking shifts of finite type as a model and subaction projections as a computing process or sofic shifts as a model and projective subdynamics as a process.

Independently, [PS10] presents some realization constructions as well as impossibility results in the weaker, yet natural case of projective subdynamics of shifts of finite type. Here, we also prove in this setting the constructability of a large class of effective shifts. To achieve this, we connect the problem to some simple notions of simulations over shifts.

Section 2 is devoted to the main definitions; Section 3 defines simulation and characterizes universality; Section 4 defines the main concept of the article, that of projective subdynamics, and recalls the known characterization in the sofic case; Section 5 introduces the intermediary notion of polyfactor, and gives a construction of it in the SFT case; Section 6 simulates it as projective subdynamics of SFT; finally Section 7 presents an independent application of the construction by proving a "Rice theorem" over projective subdynamics.

\footnotetext{
†Email: pierre.guillon@math.cnrs.fr. This article has been written mainly during a postdoctoral project supported by ECOS-Sud.
}

1365-8050 @ 2012 Discrete Mathematics and Theoretical Computer Science (DMTCS), Nancy, France 


\section{Preliminaries}

We note $\llbracket i, j \rrbracket$ the set of integers $i, i+1, \ldots, j$, and $\llbracket i, j \llbracket=\llbracket i, j-1 \rrbracket$. We also define $\mathbb{N}_{1}=\mathbb{N} \backslash\{0\}$.

Let $A$ be an alphabet (with $2 \leq|A|<\infty$ ) and $d \in \mathbb{N}_{1}$ the dimension. A shape is a subset $K \Subset \mathbb{Z}^{d}$, i.e., $K \subset \mathbb{Z}^{d}$ and $|K|<\infty$. A pattern is a finite $d$-dimensional word $u=\left(u_{i}\right)_{i \in K} \in A^{K}$, where $K \Subset \mathbb{Z}^{d}$. A configuration is an infinite one $x=\left(x_{i}\right)_{i \in \mathbb{Z}^{d}} \in A^{\mathbb{Z}^{d}}$. For any $K \subset \mathbb{Z}^{d}$ and any configuration $x \in A^{\mathbb{Z}^{d}}$, we note $x_{K}$ its restriction to $K$.

A dynamical system is a compact metric space $X$, on which some group $G$ acts continuously. The full $\mathbb{Z}^{d}$-shift on alphabet $A$ is the set of $d$-dimensional configurations $x \in A^{\mathbb{Z}^{d}}$, endowed with the product of the discrete topology, and with the action $\sigma$ of $\mathbb{Z}^{d}$ defined for any $c, i \in \mathbb{Z}^{d}$ by $\sigma^{c}(x)_{i}=x_{c+i}$. We will mainly deal with subsystems of this, i.e., closed subsets $\Sigma \subset A^{\mathbb{Z}^{d}}$ such that $\sigma^{c}(\Sigma)=\Sigma$ for any $c \in \mathbb{Z}^{d}$, which will be refered to as $\mathbb{Z}^{d}$-shifts.

Equivalently, a $\mathbb{Z}^{d}$-shift is a set $\Sigma \subset A^{\mathbb{Z}^{d}}$ of configurations defined via a collection of finite forbidden patterns $\mathcal{F}$, in the sense that $\Sigma=\left\{x \in A^{\mathbb{Z}^{d}} \mid \forall c \in \mathbb{Z}^{d}, \forall K \Subset \mathbb{Z}^{d}, \sigma^{c}(x)_{K} \notin \mathcal{F}\right\} . \Sigma$ is of finite type (SFT) if $\mathcal{F}$ can be taken finite, effective if $\mathcal{F}$ can be taken recursively enumerable.

The topological closure of the orbit $\bigcup_{c \in \mathbb{Z}^{d}} \sigma^{c}(Z)$ of $Z \subset A^{\mathbb{Z}^{d}}$ will be denoted $\bar{Z}$. For instance, in dimension 1 , a word $u$ shall be seen as a map $i \mapsto u_{i}$ from $\llbracket 0,|u| \llbracket$ to alphabet $A$. Then $\bar{\infty} u^{\infty}$ will denote the set $\left\{z \in A^{\mathbb{Z}}|\exists k \in \llbracket 0| u \mid, \llbracket, \forall j \in \mathbb{Z}, \sigma^{k+j|u|}(z)_{\llbracket 0,|u| \llbracket}=u\right\}$ of configurations periodically equal to $u$.

If $\Sigma$ is a $\mathbb{Z}$-shift over alphabet $A$, its language of shape $K \Subset \mathbb{Z}^{d}$ is the set $\mathcal{L}_{K}(\Sigma)=\left\{z_{K} \mid z \in \Sigma\right\}$ of extendable patterns for this shape. These languages completely characterize $\Sigma$; moreover, by compactness, if $\Sigma, \Lambda$ are two disjoint $\mathbb{Z}$-shifts, then there exists a finite shape $K \Subset \mathbb{Z}^{d}$ such that $\mathcal{L}_{K}(\Sigma) \cap \mathcal{L}_{K}(\Lambda)=\emptyset$.

We will actually essentially deal with $\mathbb{Z}^{2}$-shifts, but the generalization to higher dimensions is obvious.

\section{Simulations}

We define here some operations over shifts that can be seen as simulation rules, and which will help us to make constructions in the next sections. Similar compositions of operations have been recently studied in various settings [AS09, Hoc09b.

Let $X$ and $Y$ be dynamical systems corresponding to actions of the same group $\mathbb{Z}^{d}$, noted $\gamma^{c}: X \rightarrow X$ and $\delta^{c}: Y \rightarrow Y$ for $c \in \mathbb{Z}^{d}$. We note $X \succeq_{f} Y$ if there is a factor map $\Phi: X \rightarrow Y$, i.e., an onto continuous map such that $\Phi \gamma^{c}=\delta^{c} \Phi$ for any $c \in \mathbb{Z}^{d} ; Y$ is then called a factor of $X$, and if $\Phi$ is bijective, $X$ and $Y$ are called conjugate. We note $X \succeq_{i} Y$ if the action $\delta$ on $Y$ is conjugate to the action $\gamma^{k}$ on $X$ for some power $k \in \mathbb{N}_{1}^{d}$, where $\left(\gamma^{k}\right)^{c}=\gamma^{k c}$ for any $c \in \mathbb{Z}^{d}$ (and coordinatewise multiplication of vectors). We note $X \succeq_{s} Y$ if, up to conjugacy, $Y \subset X$ and $\delta$ is the restriction of $\gamma$ to $Y$.

Let $\Sigma$ and $\Gamma$ be $\mathbb{Z}^{d}$-shifts over alphabets $A$ and $B$, respectively. The previously-defined simulations can be visualized in a symbolic way. First note that $\Sigma \succeq_{i} \Gamma$ means that $\Gamma$ is essentially the bulking $\Sigma^{[K]}=\left\{\left(\sigma^{K j}(x)_{K}\right)_{j \in \mathbb{Z}^{d}} \mid x \in \Sigma\right\}$ (with coordinatewise multiplication) of $\Sigma$ for some interval product $K \Subset \mathbb{Z}^{d}$, which is a shift over alphabet $A^{K}$. It can also be seen that for any nonempty $K \Subset \mathbb{Z}^{d}, \Sigma$ is conjugate to its $K$-block representation $\Sigma^{(K)}=\left\{\left(\sigma^{i}(x)_{K}\right)_{i \in \mathbb{Z}^{d}} \mid x \in \Sigma\right\}$, which is a $\mathbb{Z}^{d}$-shift over alphabet $A^{K}$. A particular class of shift factor maps is that of parallelizations $\tilde{\Phi}: \Sigma \rightarrow \Gamma$ of alphabet projections $\Phi: A \rightarrow B$, i.e., $\tilde{\Phi}(x)_{i}=\Phi\left(x_{i}\right)$ for any $x \in \Sigma$ and $i \in \mathbb{Z}^{d}$. It is also known that $\Sigma \succeq_{f} \Gamma$ if and only if $\Gamma=\tilde{\Phi}\left(\Sigma^{(K)}\right)$ for some shape $K \Subset \mathbb{Z}^{d}$ and some parallel application $\tilde{\Phi}$ of some alphabet projection $\Phi: A^{K} \rightarrow B$. 
$\Gamma$ is called a $\mathbb{Z}^{d}$-sofic if it is a factor of a $\mathbb{Z}^{d}$-SFT. Equivalently from the last point, it is sofic if and only if it is the image of a $\mathbb{Z}^{d}$-SFT by some parallelization map. The classes of SFT, sofic shifts and effective shifts are closed under conjugacy, bulking and block representations. By the characterization above, that of sofic shifts is also closed under factor. On the contrary, $\succeq_{s}$ does not preserve any relevant property, which is why the simulation notion below will be very weak (it can be strengthened by taking the intersection with some SFT [AS09]); this will allow us to deal with a rather simple notion of universality, but will be compensated by the fact that our simulating shifts already have some structure (PSD in 9 ).

If $z: I \times J \rightarrow A$, for some intervals $I$ and $J$ of $\mathbb{Z}$ and $i \in I$, then we note $\pi_{i}(z)=\left(z_{i, j}\right)_{j \in J}$. If $I=\llbracket 0, m \llbracket$ and $J=\mathbb{Z}$, it gives a factor map $\pi_{i}$ from any $\mathbb{Z}$-shift over alphabet $A^{m}$ onto some $\mathbb{Z}$-shift over alphabet $A$. We also note $\pi_{I^{\prime}}(z)=\left(\left(z_{i, j}\right)_{i \in I^{\prime}}\right)_{j \in J}$ if $I^{\prime} \subset I$.

The product $X \times Y$ of two sets $X \in A^{\mathbb{Z}^{d}}$ and $Y \in B^{\mathbb{Z}^{d}}$ will be abusively assimilated to the set $\left\{w=\left(x_{i}, y_{i}\right)_{i \in \mathbb{Z}^{d}}\right) \in(A \times B)^{\mathbb{Z}^{d}} \mid\left(x_{i}\right)_{i \in \mathbb{Z}^{d}} \in X$ and $\left.\left(y_{i}\right)_{i \in \mathbb{Z}^{d}} \in Y\right\}$ (which is a $\mathbb{Z}^{d}$-shift if $X$ and $Y$ are). We note $X^{<1>}=X$ and $X^{<n+1>}=X^{<n>} \times X$ for $n \in \mathbb{N}$. Essentially, $X^{<n>}=$ $\left\{y \in\left(A^{n}\right)^{\mathbb{Z}^{d}} \mid \forall j \in \llbracket 0, n \llbracket, \pi_{j}(y) \in X\right\}$. We note $\Sigma \succeq_{p} \Gamma$ if $\Gamma$ is conjugate to some subshift of $\Sigma^{<n>}$ for some power $n \in \mathbb{N}$.

Each of these relations are not that interesting intrinsically, but can be associated together; the compositions will be noted $\succeq_{p s}, \succeq_{i f s}, \succeq_{p f s}$, etc...We can see, thanks to some commutation properties, that they are transitive whenever $\succeq_{i}$ and $\succeq_{p}$ are applied before $\succeq_{s}$.

We say that a $\mathbb{Z}$-shift $\Sigma$ is universal if it simulates any other $\mathbb{Z}$-shift $\Gamma$ in the sense that $\Sigma \succeq_{i s} \Gamma$. This property is easily understood in the sofic case: indeed, uncountable $\mathbb{Z}$-sofic are exactly those that have positive entropy, and they can be represented on a graph with a non-cyclic strongly connected component (equivalently, they include some infinite transitive subshift).

Proposition 1 Let $\Sigma$ be a $\mathbb{Z}$-shift. The following statements are equivalent:

1. $\Sigma$ includes some positive-entropy sofic subshift.

2. There are two words $u$ and $v$ with $u_{0} \neq v_{0},|u|=|v|$, and $\overline{\infty\{u, v\}^{\infty}} \subset \Sigma$.

3. $\Sigma \succeq_{\text {is }}\{0,1\}^{\mathbb{Z}}$.

4. $\Sigma$ is universal.

\section{Proof:}

$1 \Rightarrow 2$ If $\Sigma$ includes a positive-entropy sofic subshift, then the graph of this subshift contains a non-cyclic strongly connected component, i.e., there exists a vertex from which two arcs leave with two distinct labels, and which start paths that come back to the same vertex. Denoting $\tilde{u}, \tilde{v}$ the labels of these two paths, we can see that $u=\tilde{u}^{|\tilde{v}|}$ and $v=\tilde{v}^{|\tilde{u}|}$ satisfy the wanted conditions.

$2 \Rightarrow 3$ The $\llbracket 0,|u| \llbracket$-bulking of $\overline{\infty\{u, v\}^{\infty}}$ is a subshift that includes the full shift over alphabet $\{u, v\}$, which is essentially $\{0,1\}^{\mathbb{Z}}$.

$B \Leftrightarrow 1$ Remark that a non-trivial full shift is sofic and has positive entropy, as well as any of its iterations. 
3曰 $\Rightarrow 4$ For any $\mathbb{Z}$-shift $\Sigma$ on some alphabet $A$, we have $\{0,1\}^{\mathbb{Z}} \succeq_{i} A^{\mathbb{Z}} \succeq_{s} \Sigma$, since the letters of $A$ are in bijection with some subset of $\{0,1\}^{\lceil\log |A|\rceil}$. Hence the full shift itself is universal, and the notions of simulation are transitive.

$4 \Rightarrow 3$ This is by definition of universality.

In particular, the class of universal shifts is preserved by closing factor maps. Note that dealing with simulation $\succeq_{\text {ifs }}$ instead may widen the notion of universality to other subshifts. For our purpose though, this notion would be difficult to handle in the following.

Clock. Let $\mathcal{C}_{n}$ denote the $n$-cycle, i.e., the dynamical system $\llbracket 0, n \llbracket$ on which $\mathbb{Z}$ acts by $i \mapsto i+c \bmod n$ for any $c \in \mathbb{Z}$.

We have seen a definition of simulation that involves temporal delay, and one that involves spacial sprawl. The following lemma gives a transformation from the former to the latter.

Lemma 2 If $\Gamma$ is a $\mathbb{Z}$-shift, $A^{\mathbb{Z}}$ a full shift and $n \in \mathbb{N}$ such that $\Gamma^{[\llbracket 0, n \llbracket]} \succeq_{f s} A^{\mathbb{Z}}$, then $\mathcal{C}_{n} \times \Gamma^{<n>} \succeq_{f s} A^{\mathbb{Z}}$.

Proof: Let $\Lambda \subset \Gamma$ be closed and $\sigma^{n}$-invariant, and $\Phi: \Lambda \rightarrow A^{\mathbb{Z}}$ such that $\Phi \sigma^{n}=\sigma \Phi$. Let $\Lambda^{\prime}=$ $\left\{(i, y) \in \llbracket 0, n \llbracket \times \Gamma^{<n>} \mid \forall j \in \llbracket 0, n \llbracket, \pi_{j}(y) \in \sigma^{j-i \bmod n}(\Gamma)\right\}$.

$$
\begin{aligned}
\Psi: \Lambda^{\prime} & \rightarrow A^{\mathbb{Z}} \\
(i, y) & \mapsto\left(\Phi\left(\sigma^{j}\left(\pi_{i+j \bmod n}(y)\right)\right)_{0}\right)_{j \in \mathbb{Z}}
\end{aligned}
$$

is also a factor map, since for any $(i, y) \in \Gamma^{\prime}$, we have:

$$
\tilde{\Phi}(i+1 \bmod n, \sigma(y))=\left(\Phi\left(\sigma^{j+1}\left(\pi_{i+1+j \bmod n}(y)\right)\right)_{0}\right)_{j \in \mathbb{Z}}=\sigma\left(\tilde{\Phi}\left(i, y_{0}, \ldots, y_{n-1}\right)\right) .
$$

Moreover, $\Psi$ is onto $A^{\mathbb{Z}}$ since, if $z \in A^{\mathbb{Z}}$, for $0 \leq i<n$, the surjectivity of $\Phi$ and $\sigma$ gives some $y^{i} \in Y$ such that $\Phi\left(\sigma^{n}\left(y^{i}\right)\right)=\left(z_{n j+i}\right)_{j \in \mathbb{Z}}$. By construction, for any $j \in \mathbb{Z}$ and any $i \in \llbracket 0, n \llbracket$, we have:

$$
\Psi\left(0, \sigma^{n}\left(y^{0}\right), \sigma^{n-1}\left(y^{1}\right), \ldots, \sigma\left(y^{n-1}\right)\right)_{n j+i}=\Phi\left(\sigma^{n j+i}\left(\sigma^{n-i}\left(y^{i}\right)\right)\right)_{0}=\sigma^{j+1}\left(\Phi\left(y^{i}\right)\right)_{0}=z_{n j+i} .
$$

Proposition 3 If $\Sigma$ is a $\mathbb{Z}$-shift, the following are also equivalent to universality (and to properties of Proposition [1]:

5. $\Sigma \succeq_{p s}\{0,1\}^{\mathbb{Z}}$.

6. For any subshift $\Gamma, \Sigma \succeq_{p s} \Gamma$.

\section{Proof:}

$6 \Rightarrow 5 \Rightarrow 1$ Positive entropy is preserved by product and supersystem, i.e., the entropy of a system is more than that of any of its subsystems.

5 $\Rightarrow 6$ It is clear that every subshift can essentially be seen on an alphabet of the form $\{0,1\}^{n}$ with $n \in \mathbb{N}$, that is to say as being included in $\left(\{0,1\}^{\mathbb{Z}}\right)^{<n>}$. 
3 $\& 2 \Rightarrow 5$ One can verify that if $u_{0} \neq v_{0}$ and $|u|=|v|=n$, then ${ }^{\infty}(u u v)^{\infty}$ is a word of smallest period $3 n$, and that $\overline{\infty(u u v)^{\infty}}$ is then conjugate to $\mathcal{C}_{3 n}$, which factors onto $\mathcal{C}_{n}$. It is also clear that simulations are compatible with the product of systems. It results that $\overline{\infty(u u v)^{\infty}} \times \Gamma^{<n>} \succeq_{f} \mathcal{C}_{n} \times \Gamma^{<n>} \succeq_{f s}$ $\{0,1\}^{\mathbb{Z}}$ by Lemma 2 and, by hypothesis, $\overline{\infty(u u v)^{\infty}} \subset \Gamma$, which gives $\Gamma^{<n+1>} \succeq_{f s}\{0,1\}^{\mathbb{Z}}$.

\section{Projective subdynamics}

If $y=\left(y_{k, i}\right)_{k, i \in \mathbb{Z}^{2}} \in A^{\mathbb{Z}^{2}}$ is a configuration and $k \in \mathbb{Z}$, then $\tau^{k}(y)=\left(y_{k, i}\right)_{i \in \mathbb{Z}}$ will denote the projected $k^{\text {th }}$ column. We note $\tau=\tau^{0}$. The projective subdynamics (PSD) of some $\mathbb{Z}^{2}$-shift $X$ is the $\mathbb{Z}$-shift $\tau(X)$. The (vertical) subaction is the dynamical system where $X$ is seen as being acted on by the restriction of $\sigma$ to the subgroup $\{0\} \times \mathbb{Z}$ (only shifting vertically). Note that the PSD is a factor of the subaction by the map $\Phi$ defined by $\Phi(x)_{i}=x_{0, i}$ for $x \in X$ and $i \in \mathbb{Z}$.

The notions of PSD and subactions can of course be defined with respect to any dimension and any direction (subgroups of $\mathbb{Z}^{d}$ ), and all the following results will be adaptable in the general setting, but, for the sake of clarity, we will stick to the simple case of columns in bidimensional configurations.

If $\Sigma \subset A^{\mathbb{Z}}$ is a $\mathbb{Z}$-shift, let $\Sigma^{\mathbb{Z}}$ denote the $\mathbb{Z}^{2}$-shift $\left\{x \in A^{\mathbb{Z}^{2}} \mid \forall j \in \mathbb{Z}, \tau^{j}(x) \in \Sigma\right\}$. Remark that if $\Sigma$ is an SFT, then so is $\Sigma^{\mathbb{Z}}$. If $\Phi$ is a factor map between the $\mathbb{Z}$-shifts $\Lambda$ and $\Sigma$, then we can define a parallelization $\tilde{\Phi}$ from $\Lambda^{\mathbb{Z}}$ onto $\Sigma^{\mathbb{Z}}$ such that $\tau^{k}(\tilde{\Phi}(x))=\Phi\left(\tau^{k}(x)\right)$ for any $x \in \Lambda^{\mathbb{Z}}$ and any $k \in \mathbb{Z}$.

In the following sections, we will be interested in the PSD of $\mathbb{Z}^{2}$-SFT.

Proposition 4 The class of PSD of $\mathbb{Z}^{2}$-SFT is invariant by product, conjugacy, and by SFT factor preimages, i.e., if $\Phi$ is a factor map from a $\mathbb{Z}$-SFT $\Sigma$ onto a $\mathbb{Z}$-shift $\Lambda$ and $X$ a $\mathbb{Z}^{2}$-SFT with $\tau(X) \subset \Lambda$, then $\Phi^{-1}(\tau(X))$ is the PSD of some $\mathbb{Z}^{2}$-SFT.

\section{Proof:}

- Clearly, the PSD of a product $\mathbb{Z}^{2}$-shift is the product $\mathbb{Z}$-shift of their two PSD.

- Assume that $\Phi$ is a conjugacy between a $\mathbb{Z}$-shift $\Sigma$ and $\tau(X)$ for some $\mathbb{Z}^{2}$-SFT $X$, and $\tilde{\Phi}: \Sigma^{\mathbb{Z}} \rightarrow \Lambda^{\mathbb{Z}}$ its parallelization such that $\forall x \in \Sigma^{\mathbb{Z}}, \tau(\tilde{\Phi}(x))=\Phi(\tau(x))$. It is clear that $\tilde{\Phi}$ is a conjugacy between $\Sigma^{\mathbb{Z}}$ and $\tau(X)^{\mathbb{Z}}$, and that $Y=\tilde{\Phi}^{-1}(X)$ is a $\mathbb{Z}^{2}$-SFT with $\tau(Y)=\Phi^{-1}(\tau(X)=\Sigma$.

- Let $\Phi$ be as in the statement. As above, its parallelization $\tilde{\Phi}: \Sigma^{\mathbb{Z}} \rightarrow \Lambda^{\mathbb{Z}}$ satisfies that the preimage $Y=\tilde{\Phi}^{-1}(X)$ is a $\mathbb{Z}^{2}$-SFT, since $\Sigma^{\mathbb{Z}}$ and $X$ both are; by construction, $\tau(Y)=\Phi^{-1}(\tau(X))$.

Before dealing further with the PSD of $\mathbb{Z}^{2}$-SFT, let us state what is known about PSD of $\mathbb{Z}^{2}$-sofic.

Proposition 5 Let $\Sigma$ be a $\mathbb{Z}$-shift. The following are equivalent.

1. $\Sigma$ is the PSD of some $\mathbb{Z}^{2}$-sofic.

2. $\Sigma$ is a factor of the PSD of some $\mathbb{Z}^{2}-S F T$.

3. $\Sigma$ is the factor of the subaction of some $\mathbb{Z}^{2}-S F T$. 
Proof:

1 $\Rightarrow 2$ If $\Sigma=\tau(\tilde{\Phi}(X)) \subset A^{\mathbb{Z}}$ for some $\mathbb{Z}^{2}$-SFT $X$ over alphabet $B$ and some factor map $\tilde{\Phi}$ based on an alphabet projection $\Phi: B \rightarrow A$, then $\Sigma=\tilde{\Phi}(\tau(X))$ if we define $\tilde{\Phi}(x)_{i}=\Phi\left(x_{i}\right)$ for any $i \in \mathbb{Z}$.

2 $\Rightarrow 1$ Assume $\Sigma=\Phi(\tau(X))$ for some factor map $\Phi$ and some $\mathbb{Z}^{2}$-SFT $X$. Then the parallelization $\tilde{\Phi}: \tau(X)^{\mathbb{Z}} \rightarrow \Sigma^{\mathbb{Z}}$ is such that $\tau(\tilde{\Phi}(X))=\Phi(\tau(X))=\Sigma$, and $\tilde{\Phi}(X)$ is sofic as a factor of a $\mathbb{Z}^{2}$-SFT.

$2 \Rightarrow 3$ The PSD is a factor of the subaction, and the relation of factor is transitive.

$3 \Leftrightarrow 2$ Let $\Phi$ be a factor map from the vertical subaction of $X$ onto $\Sigma$ for some $\mathbb{Z}^{2}$-SFT $X$. By a standard uniform-continuity argument, some block representation of $X$ will (while still being SFT) transform $\Phi$ into a simple projection to the central cell.

As a consequence of this, the class of PSD of $\mathbb{Z}^{2}$-sofic is invariant by factor; it actually admits the following elegant characterization.

Let us define for any $\mathbb{Z}$-configuration $x \in A^{\mathbb{Z}}$ the $\mathbb{Z}^{2}$-configuration $\mathcal{A}(x)$ by $\tau^{j}(\mathcal{A}(x))=x$, for any $j \in \mathbb{Z}$. If $\Sigma$ is a $\mathbb{Z}$-shift over alphabet $A$, then $\mathcal{A}(\Sigma)$ is a $\mathbb{Z}^{2}$-shift.

Theorem 6 ([DRS10, AS10]) The following are equivalent (and are thus also equivalent to the statements in Proposition 5).

4. $\Sigma$ is effective.

5. $\mathcal{A}(\Sigma)$ is sofic.

The problem of finding a similar characterization of $\mathbb{Z}$-shifts that can be obtained as PSD of $\mathbb{Z}^{2}$-SFT remains. It is clear that this class contains all $\mathbb{Z}$-SFT. In [CFG10], some constructions are given of cellular automata defined over $\mathbb{Z}$-SFT and that have specific ultimate traces, which actually give projective subdynamics of some $\mathbb{Z}^{2}$-SFT: in particular, all positive-entropy sofic subshifts can be obtained that way. On the other hand, [GR10] gives some impossibility results in that particular subsetting. In [PS10], both more constructions and impossibility results are presented, in the general setting. In particular, a full characterization of $\mathbb{Z}$-sofic PSD of $\mathbb{Z}^{2}$-SFT is given, emphasizing moreover on the difference between the so-called stable and unstable PSD. Note that the $\mathbb{Z}^{2}$-SFT can realize strictly fewer $\mathbb{Z}$-shifts than $\mathbb{Z}^{2}$-sofic do. There are even $\mathbb{Z}$-sofic that are not realizable as PSD of $\mathbb{Z}^{2}$-SFT, such as the shift of the configurations having state 0 everywhere except for at most one cell. The next two sections are devoted to realizing some class of $\mathbb{Z}$-shifts that goes further than the sofic case.

\section{Polyfactors}

The polyfactor of some $\mathbb{Z}^{2}$-shift $X$ over alphabet $A^{m}$, with $m \in \mathbb{N}_{1}$, is the union $\stackrel{\circ}{\tau}(X)=$ $\bigcup_{0 \leq i<m} \pi_{i}(\tau(X))$, which can be seen as the projective subdynamics of some system which is invariant by some powers of the shift, but not by the whole action (periodic local constraints). Note that the notion of polyfactor depends on the interpretation of the alphabet as a power of another alphabet. In particular, the projective subdynamics of some shift is also its polyfactor (if we interpret $m=1$ ).

Let us see conditions on the subshift that allow it to be the polyfactor of some SFT. 
Lemma 7 If $\Sigma$ is a $\mathbb{Z}$-shift and $X$ a $\mathbb{Z}^{2}$-shift such that $\Sigma \succeq_{p s} \tau(X) \succeq_{f} \Sigma$, then $\Sigma$ is the polyfactor of some $\mathbb{Z}^{2}$-shift $Y$ conjugate to $X$.

Proof: Let $n \in \mathbb{N}$ be such that $\tau(X) \subset \Sigma^{<n>}, \Psi$ be a factor map from $\tau(X)$ onto $\Sigma$, and $\tilde{\Psi}$ its parallelization, i.e., $\forall x \in X, \tau(\tilde{\Psi}(x))=\Psi(\tau(x))$. The product $Y=\tilde{\Psi}(X) \times X$ is conjugate to $X$ (they are linked by maps $\Psi \times$ id and $\pi_{1}$ ). Moreover, it can be seen as a $\mathbb{Z}^{2}$-shift with $1+n$ columns; the first one is equal to $\Sigma$, and the other $n$ are included in it.

The interest of introducing polyfactors of $\mathbb{Z}^{2}$-SFT is that their class is more robust than that of their projective subdynamics, as illustrated by the following remarks (all of which are not useful for our main construction).

Proposition 8 The class of polyfactors of $\mathbb{Z}^{2}$-SFT is invariant by projection union (if the alphabet is a power), product, union, conjugacy, weak iteration (reading every n letters), and by SFT factor preimages (see Proposition 4).

Proof:

- If $\Sigma=\stackrel{\circ}{\tau}(X)$ where $X$ is a $\mathbb{Z}^{2}$-SFT over alphabet $\left(A^{m}\right)^{n}$, with $m, n \in \mathbb{N}_{1}$, then $\bigcup_{0 \leq i<m} \pi_{i}(\Sigma)$ is also the polyfactor of $X$, seen as a $\mathbb{Z}^{2}$-SFT over alphabet $A^{m n}$.

- Assume $\Sigma=\stackrel{\circ}{\tau}(X) \subset A^{\mathbb{Z}}$ and $\Gamma=\stackrel{\circ}{\tau}(Y) \subset B^{\mathbb{Z}}$, i.e., there are $m, n \in \mathbb{N}$ such that $\tau(X) \subset \Sigma^{<m>}$ and $\tau(Y) \subset \Gamma^{<m>}$. Then $\Sigma \times \Gamma$ can be seen as the polyfactor of the $\mathbb{Z}^{2}$-shift $\left\{z \mid \exists x \in \Sigma, y \in \Gamma, \forall i \in \llbracket 0, m \llbracket, \forall j \in \llbracket 0, n \llbracket, \pi_{i+j m}(z)=\left(\pi_{i}(x), \pi_{j}(m)\right\}\right.$ over alphabet $(A \times B)^{m n}$.

- The previous two points give the union.

- If a $\mathbb{Z}$-shift $\Sigma$ over alphabet $A$ is conjugate to $\stackrel{\circ}{\tau}(X)$ for some $\mathbb{Z}^{2}$-SFT over alphabet $A^{m}$ for some $m \in \mathbb{N}$, then it is clear that this conjugacy can be parallelized into a conjugacy $\tilde{\Phi}: \Sigma^{<m>} \rightarrow \stackrel{\circ}{\tau}$ $(X)^{<m>}$; by Proposition 4 , $\tilde{\Phi}^{-1}(\tau(X))$ can be obtained as $\tau(Y)$ for some $\mathbb{Z}^{2}$-SFT $Y$. One can see that $\stackrel{\circ}{\tau}(Y)=\bigcup_{0 \leq i<m} \pi_{i}\left(\tilde{\Phi}^{-1}(\tau(X))\right)=\bigcup_{0 \leq i<m} \Phi^{-1}\left(\pi_{i}(\tau(X))\right)=\Phi^{-1}(\stackrel{\circ}{\tau}(X))=\Sigma$.

- Invariance by SFT factor preimage comes from 4 in the same way as conjugacy.

- If $K \Subset \mathbb{Z}^{d}$ and $X$ is a $\mathbb{Z}^{2}$-SFT, then the bulking $X^{[K]}$ is one also; its polyfactor $\stackrel{\circ}{\tau}\left(X^{[K]}\right)$ consists exactly of all weak iterations of $\stackrel{\circ}{\tau}(X)$.

Proposition 9 If $\Sigma \succeq_{p s} \Lambda \succeq_{f s} \tau(X) \succeq_{f} \Sigma$ for some $\mathbb{Z}$-SFT $\Lambda$ and some $\mathbb{Z}^{2}$-SFT $X$, then $\Sigma$ is the polyfactor of some $\mathbb{Z}^{2}$-SFT.

Proof: Let $n \in \mathbb{N}$ be such that $\Lambda \subset \Sigma^{<n>}, \Gamma \subset \Lambda$ and $\Phi: \Gamma \rightarrow \tau(X)$ a factor map, which can actually be extended to $\Lambda$. Then $\Phi^{-1}(\tau(X))$ is the PSD of some $\mathbb{Z}^{2}$-SFT $Y$ thanks to Proposition 4 . We obtain $\Sigma \succeq_{p s} \Lambda \succeq_{s} \tau(Y)$ and $\tau(Y) \succeq_{f} \tau(X) \succeq_{f} \Sigma$, which gives $\Sigma \succeq_{p s} \tau(Y) \succeq_{f} \Sigma$; Lemma 7 allows to conclude. 
Corollary 10 If $\Sigma$ is an effective $\mathbb{Z}$-shift and contains some positive-entropy sofic subshift, then it is the polyfactor of some $\mathbb{Z}^{2}$-SFT.

Proof: It is enough to use Proposition 9 with $\Lambda$ some full shift simulated by $\Sigma$ (see Proposition 1 ) and $X$ some $\mathbb{Z}^{2}$-sofic whose projective subdynamics is $\Sigma$ (see Theorem 6 ).

\section{Projective subdynamics of SFT}

Let us see a construction that turns the polyfactor of some SFT into the projective subdynamics of a modified SFT.

Consider a $\mathbb{Z}$-shift $S$ over alphabet $A^{m}$ for some $m \in \mathbb{N}$. $S$ is marking if $S_{0} \cap S_{i}=\emptyset$ for $0<i<m$ and $S_{i}=\left\{w \in\left(A^{2 m-1}\right)^{\mathbb{Z}} \mid \pi_{\llbracket i, i+m \llbracket}(w) \in S\right\}$. By compactness, we have that $S$ is marking if and only if there exists some length $l \in \mathbb{N}$ such that the languages $\mathcal{L}_{\llbracket 0, l \llbracket}\left(S_{i}\right)$ are pairwise disjoint for $i \in \llbracket 0, m \llbracket$.

This definition can be seen as the impossibility to interpret a two-dimensional configuration into two distinct juxtapositions of stripes of $S$. Of course, any subshift of a marking shift is also marking.

Here are two classes of examples of marking shifts.

Example 11 If $\Gamma$ and $\Lambda$ are two disjoint $\mathbb{Z}$-shifts over alphabet $A$ and $m \in \mathbb{N}$, then the following set is marking:

$$
S_{\Gamma, \Lambda}^{m}=\left\{w \in\left(A^{2 m+2}\right)^{\mathbb{Z}} \mid \forall i \in \llbracket m, 2 m \rrbracket, \pi_{i}(w) \in \Gamma \text { and } \pi_{2 m+1}(w) \in \Lambda\right\} .
$$

Example 12 If $u$ and $v$ are two distinct words with same length over alphabet $A$ and $m \in \mathbb{N}$, then the following set is marking:

$S_{u, v}^{m}=\left\{w \in\left(A^{2 m+2}\right)^{\mathbb{Z}}|\exists j \in \llbracket 0| u \mid, \rrbracket, \forall i \in \llbracket m, 2 m \rrbracket, \pi_{i}(w)=\sigma^{j}\left({ }^{\infty} u^{\infty}\right)\right.$ and $\left.\pi_{2 m+1}(w)=\sigma^{j}\left({ }^{\infty} v^{\infty}\right)\right\}$.

For $x \in\left(A^{m}\right)^{\mathbb{Z}^{2}}$, we define the m-unbulking of $x$ with shift $i \in \mathbb{Z}$ as the configuration $y=\bigotimes_{m}^{i}(x)$

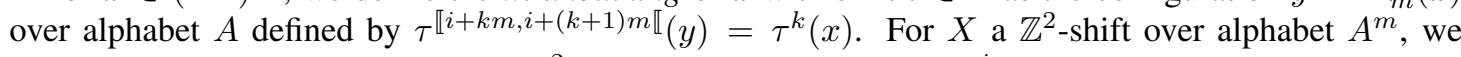
define the m-unbulking of $X$ as the $\mathbb{Z}^{2}$-shift $\bigotimes_{m}(X)=\bigcup_{i \in \llbracket 0, m \llbracket} \bigotimes_{m}^{i}(X)$ over alphabet $A$. It flattens the configurations by alternating the layers (like the contrary of a $\llbracket 0, m \llbracket \times\{0\}$-bulking). In particular, $\tau\left(\otimes_{m}(X)\right)=\stackrel{\circ}{\tau}(X)$. If $X$ is an SFT, then $\otimes_{m}(X)$ need not be so, but this is where marking shifts is useful.

Lemma 13 Let $X$ be a $\mathbb{Z}^{2}$-SFT over alphabet $A^{m}$ for some $m \in \mathbb{N}$, such that $\tau(X)$ is marking. Then $\bigotimes_{m}(X)$ is a $\mathbb{Z}^{2}$-SFT over alphabet $A$, and $\tau\left(\bigotimes_{m}(X)\right)=\stackrel{\circ}{\tau}(X)$. Moreover, its local constraints can be effectively computed from that of $X$.

Proof: Since the $S_{i}=\left\{w \in\left(A^{2 m-1}\right)^{\mathbb{Z}} \mid \pi_{\llbracket i, i+m \llbracket}(w) \in \tau(X)\right\}$ are disjoint for $i \in \llbracket 0, m \llbracket$, by compactness they actually differ on patterns of a bounded height. Hence some local constraints can impose patterns of this height to cycle through the $S_{i}$. Moreover, for each $i$, it is easy to check locally the constraints of $X$ (that may have a larger range) with respect to this unique interpretation.

Lemma 14 Let $X$ be a $\mathbb{Z}^{2}$-SFT over alphabet $A^{m}$, and $Y, Z$ two nonempty $\mathbb{Z}^{2}$-SFT over alphabet $A$ such that $\tau(Y) \cap \tau(Z)=\emptyset$. Then $X^{\prime}=\bigotimes_{2 m+2}\left(X \times Y^{<m+1>} \times Z\right)$ is a $\mathbb{Z}^{2}$-SFT. It is empty if $X$ is, otherwise $\tau\left(X^{\prime}\right)=\stackrel{\circ}{\tau}(X) \cup \tau(Y) \cup \tau(Z)$. Moreover, its local constraints can be effectively computed from that of $X, Y, Z$. 
Proof: The PSD $\tau\left(X^{\prime}\right)$ is included in $S_{\tau(Y), \tau(Z)}^{m}$, which is marking by Example 11. Hence Lemma 13 gives the result. It is clear that everything is effective.

Proposition 15 Let $\Sigma$ be the polyfactor of some $\mathbb{Z}^{2}$-SFT $X$ over alphabet $A^{m}$ for some $m \in \mathbb{N}_{1}$, and $Y, Z$ two nonempty $\mathbb{Z}^{2}$-SFT over alphabet $A$ such that $\tau(Y) \cap \tau(Z)=\emptyset$. Then $\Sigma \cup \tau(Y) \cup \tau(Z)$ is the PSD of some $\mathbb{Z}^{2}$-SFT over A.

Proof: If $\Sigma \neq \emptyset$, then Lemma 14 gives the result; otherwise we can apply the same lemma while fixing $m=0$.

The interesting case will actually be when $\tau(Y)$ and $\tau(Z)$ are contained in $\Sigma$.

Theorem 16 Any effective $\mathbb{Z}$-shift including some positive-entropy sofic subshift is the PSD of some $\mathbb{Z}^{2}$ SFT.

Proof: This directly comes from Proposition 15, Corollary 10, and the fact that any positive-entropy $\mathbb{Z}$-sofic contains two disjoint periodic orbits, which are trivially realizable as PSD of periodic $\mathbb{Z}^{2}$-SFT.

Any effective universal $\mathbb{Z}$-shift is then realizable in that sense, and note that their class is preserved by closing maps.

Another consequence of this construction is the following.

Corollary 17 Let $\left(X_{i}\right)_{0 \leq i<m}$ be a finite family of $\mathbb{Z}^{2}$-SFT among which two have disjoint PSD, say $\tau\left(X_{0}\right) \cap \tau\left(X_{1}\right)=\emptyset$. Then $\bigcup_{0 \leq i<m} \tau\left(X_{i}\right)$ is the PSD of some $\mathbb{Z}^{2}$-SFT.

Proof: By Proposition $8 \bigcup_{2 \leq i<m} \tau\left(X_{i}\right)$ is the polyfactor of some $\mathbb{Z}^{2}$-SFT. Then Proposition 15 gives the result.

The simple cases of application of this corollary are in the case of two PSD which are either disjoint or contain two distinct periodic orbits. We can be more precise: by using Example 12 in Lemma 14, we can reprove [PS10, Proposition 5.3]: if $\left(X_{i}\right)_{0 \leq i<m}$ is a finite family of $\mathbb{Z}^{2}$-SFT such that $\Sigma=\bigcup_{0 \leq i<m} \tau\left(X_{i}\right)$ contains two distinct periodic configurations, then $\Sigma$ is the PSD of some $\mathbb{Z}^{2}$-SFT. This is not a direct corollary of the previous statement, since the two distinct periodic configurations could here be in the same non-uniform periodic orbit.

\section{Undecidability}

The transformation of polyfactors into projective subdynamics allows the following theorem à la Rice. This is largely inspired by [CG07, CFG10], but note that it is not a direct corollary of the corresponding result on traces of cellular automata, since we deal here with more non-trivial properties.

Theorem 18 For any property $\mathcal{P}$ satisfied by the PSD of some $\mathbb{Z}^{2}$-SFT over alphabet $\{0,1\}$, but not all of them, the following problem is undecidable:

Input: $a \mathbb{Z}^{2}$-SFT over alphabet $\{0,1\}$.

Problem: $\tau(X) \in \mathcal{P}$ ? 
Proof: Assume that the full shift $\{0,1\}^{\mathbb{Z}}$ satisfies $\mathcal{P}$ (otherwise consider the complement of $\mathcal{P}$ ); let $Y$ be some $\mathbb{Z}^{2}$-SFT such that $\tau(Y)$ does not satisfy $\mathcal{P}$, and $w \in\{0,1\}^{\llbracket 0, l \mathbb{I}^{2}}$ a forbidden pattern for $Y$, with $l \in \mathbb{N}$. We can consider the (periodic) $\mathbb{Z}$-SFT ${ }^{\infty} w^{\infty}$ over alphabet $A^{l}$, as a periodic vertical superposition of these blocks $w$. Let us prove that, if the problem above was decidable, then we could decide the emptiness of binary $\mathbb{Z}^{2}$-shifts. Indeed, let us be given an arbitrary $\mathbb{Z}^{2}$-shift $X$ over alphabet $\{0,1\}$. We can compute the $\mathbb{Z}^{2}$-SFT $X^{\prime}=\left(\bar{\infty} w^{\infty}\right)^{\mathbb{Z}} \times X \times\{0,1\}^{\mathbb{Z}^{2}}$, that we must see over alphabet $\{0,1\}^{l+2}$, with $l$ layers representing periodic superpositions of blocks $w$, a layer representing $X$ and a layer with a full shift. Then from Lemma 14 we can compute the $\mathbb{Z}^{2}$-SFT $X^{\prime \prime}=\bigotimes_{2 l+6}\left(X^{\prime} \times\left\{{ }^{\infty} 0^{\infty}\right\}^{<l+3>} \times\left\{{ }^{\infty} 1^{\infty}\right\}\right)$. Now, since pattern $w$ appears periodically in configurations of $X^{\prime \prime}$, we have that $Y^{\prime}=Y \sqcup X^{\prime \prime}$ is still a $\mathbb{Z}^{2}$-SFT. If $X$ is empty, then so is $X^{\prime}$, and so is $X^{\prime \prime}$, hence $Y^{\prime}=Y$ and $\tau\left(Y^{\prime}\right)=\tau(Y) \notin \mathcal{P}$. Otherwise, $\tau\left(Y^{\prime}\right) \supset \tau\left(X^{\prime \prime}\right) \supset \stackrel{\circ}{\tau}\left(X^{\prime}\right) \supset\{0,1\}^{\mathbb{Z}}$, hence $\tau\left(Y^{\prime}\right)=\{0,1\}^{\mathbb{Z}} \in \mathcal{P}$. As a consequence, if we could decide whether the PSD of the $\mathbb{Z}^{2}$-SFT $Y$ satisfied $\mathcal{P}$ or not, then we would be able to decide whether $X$ is empty. Yet, this problem is known to be undecidable (see [Ber66] for a proof on Wang tile model, which can easily be simulated effectively by binary $\mathbb{Z}^{2}$-SFT).

Taking $Y$ sofic instead of SFT allows the same statement for $\mathbb{Z}^{2}$-sofic.

\section{Conclusion}

We have presented a construction of SFT that have a given PSD among a large class. It is clear that it could be adapted to PSD corresponding to dimensions higher than 2, codimensions higher than 1, and other subgroups than vertical columns.

However, this construction leaves as open problems a general characterization of PSD of SFT. Some effective positive-entropy shifts may not include positive-entropy sofic subshifts. A difficult case is also the case of null-entropy shifts. It was well understood by [PS10] in the sofic case; this construction can be thought of as some kind of simulation, and maybe Proposition 9 could involve simulations performed by non-universal shifts. Impossibility results are also lacking outside the sofic one-dimensional case [PS10].

What could be interesting too is to study the case of deterministic SFT (or, equivalently, with some given expansiveness directions). But it is already difficult to understand the case of deterministic sofic (for instance whether the construction of [AS10] could be "determinized"). Cellular automata (which correspond to deterministic SFT with additional regularity properties, or seen as actions of $\mathbb{N} \times \mathbb{Z}$ rather than $\mathbb{Z}^{2}$ ) have been the subject of independent works, still far from characterizations, be it the limit set (PSD orthogonal to the expansiveness direction) [Hur87, Maa95] or the trace (parallel) [CFG07, CFG10].

Another question was asked by E. Jeandel and R. Pavlov [Pav10, Question 2]: in a similar flavor to Theorem 6, what can we say about the class of $\mathbb{Z}$-shifts $\Sigma$ such that $\Sigma^{\mathbb{Z}}$ is a $\mathbb{Z}^{2}$-sofic? It is clear that $\Sigma$ is SFT if and only if $\Sigma^{\mathbb{Z}}$ is, showing that this kind of dimension increase leaves much less freedom than $\mathcal{A}$. It seems there are no example of non-sofic $\Sigma$ with $\Sigma^{\mathbb{Z}}$ sofic. 


\section{References}

[AS09] Nathalie Aubrun and Mathieu Sablik. An order on sets of tilings corresponding to an order on languages. In Susanne Albers and Jean-Yves Marion, editors, $26^{\text {th }}$ International Symposium on Theoretical Aspects of Computer Science (STACS'09), Freiburg, Germany, February 2009. IBFI Schloss Dagstuhl.

[AS10] Nathalie Aubrun and Mathieu Sablik. Simulation of effective subshifts by two-dimensional sft and a generalization. preprint, 2010 .

[Ber66] Robert Berger. The undecidability of the domino problem. Memoirs of the American Mathematical Society, 66:72, 1966.

[CFG07] Julien Cervelle, Enrico Formenti, and Pierre Guillon. Sofic trace of a cellular automaton. In S. Barry Cooper, Benedikt Löwe, and Andrea Sorbi, editors, Computation and Logic in the Real World, $3^{\text {rd }}$ Conference on Computability in Europe (CiE07), volume 4497 of Lecture Notes in Computer Science, pages 152-161, Siena, Italy, June 2007. Springer-Verlag.

[CFG10] Julien Cervelle, Enrico Formenti, and Pierre Guillon. Ultimate traces of cellular automata. In Jean-Yves Marion, editor, $27^{\text {th }}$ International Symposium on Theoretical Aspects of Computer Science (STACS'10), Nancy, France, March 2010.

[CG07] Julien Cervelle and Pierre Guillon. Towards a Rice theorem on traces of cellular automata. In Ludek Kučera and Antonín Kučera, editors, $32^{\text {nd }}$ International Symposium on the Mathematical Foundations of Computer Science, volume 4708 of Lecture Notes in Computer Science, pages 310-319, Český Krumlov, Czech Republic, August 2007. Springer-Verlag.

[DKB05] Jean-Charles Delvenne, Petr Kůrka, and Vincent Blondel. Decidability and universality in symbolic dynamical systems. Fundamenta Informatica, XX:1-25, 2005.

[DRS10] Bruno Durand, Andrei Romashchenko, and Alexander Shen. Fixed-point tile sets and their applications. draft, September 2010.

[GR10] Pierre Guillon and Gaétan Richard. Asymptotic behavior of dynamical systems. preprint, April 2010.

[Hoc09a] Michael Hochman. On the dynamics and recursive properties of multidimensional symbolic systems. Inventiones Mathematica, 176(1):131-167, April 2009.

[Hoc09b] Michael Hochman. On universality in multidimensional symbolic dynamics. Discrete \& Continuous Dynamical Systems, 2(2), 2009.

[Hur87] Lyman P. Hurd. Formal language characterizations of cellular automaton limit sets. Complex Systems, 1:69-80, 1987.

[Kůr97] Petr Kůrka. Languages, equicontinuity and attractors in cellular automata. Ergodic Theory \& Dynamical Systems, 17(2):417-433, April 1997.

[Maa95] Alejandro Maass. On the sofic limit set of cellular automata. Ergodic Theory \& Dynamical Systems, 15:663-684, 1995.

[Pav10] Ronnie Pavlov. A class of nonsofic $\mathbb{Z}^{d}$ shift spaces. preprint, 2010.

[PS10] Ronnie Pavlov and Michael Schraudner. Classification of sofic projective subdynamics of multidimensional shifts of finite type. preprint, 2010. 
